\title{
A New Topology of AC-AC Ground Power Unit for Aeronautical Applications
}

\author{
M. Rivera, D. Faúndez, J. Kolar and P. Wheeler
}

\begin{abstract}
Nowadays, electrical energy is the alternative to supply aircraft systems. In this paper a new converter for ground power units supply in aircraft applications is proposed. The topology consists in a rectifier with an active current injection circuit linked to a three level neutral point clamped converter. The design procedure is detailed in this paper showing the principal requirements for the safe operation of the converter. Simulation results validate the good performance of the proposed system.
\end{abstract}

Index Terms-Multilevel converters, neutral point converters, control techniques

\section{INTRODUCCIÓN}

En la actualidad es preferible usar energía eléctrica para reemplazar los medios neumáticos, hidráulicos y mecánicos para alimentar los sistemas de aviones tales como funciones de utilidad, actuadores de control de vuelo y el sistema de control de aire acondicionado. Esta opción presenta los beneficios de menor peso de la aeronave, reduce la necesidad de equipo de apoyo en tierra, así como mantenimiento y confiabilidad expandida. Las unidades de potencia de tierra (GPU) son consideradas como fuente de alimentación para aeronaves en aviación militar y civil, barcos, radares y otros. La maquinaria está destinada a establecerse en los aeropuertos y utilizarse como fuente de alimentación eléctrica para aviones durante las operaciones de reposición de combustible y carga. Esto es preferible con el objetivo de reducir costos, porque la energía puede ser convertida en el suelo de una manera barata y eficiente en vez de la quema de combustible del avión. La principal aplicación de este equipo es apagar la unidad de potencia auxiliar (APU) del avión para disminuir la contaminación del aire, el ruido, la revisión de mantenimiento de la APU o motor de arranque inclusivo. Los principales desafíos para estos sistemas son:

- Proporcionar 115[V]@400[Hz] independientemente de la carga.

- Minimizar el impacto de la corriente de entrada en el sistema eléctrico de la aeronave, deben ser con contenidos armónicos reducidos.

- Para minimizar los requisitos de potencia reactiva, se debe lograr un factor de potencia de entrada alto.

- Maximizar la densidad de potencia para reducir el peso y el tamaño.

M. Rivera and D. Faúndez are with the Faculty of Engineering, Universidad de Talca, Curico, Chile. (e-mail: marcoriv@utalca.cl, dfaundezcorrea@gmail.com)

J. Kolar is with the Power Electronics Systems Laboratory, Dept. of Information Technology and Electrical Engineering, ETH Zurich, Zurich, Switzerland. (e-mail: kolar@lem.ee.ethz.ch.

P. Wheeler is with the Faculty of Engineering, The University of Nottingham, Nottingham, UK. (e-mail: Pat.Wheeler@nottingham.ac.uk)
Al principio, las unidades de energía de tierra utilizaban técnicas de modulación optimizadas que eliminaban o minimizaban la distorsión armónica total y, por lo tanto, disminuyendo el tamaño de los filtros de salida. Como se analizó anteriormente, varios inversores que operan a una frecuencia de conmutación reducida se incluyen a través de ciertos transformadores para producir una tensión de salida de 12, 24 o incluso 48 pulsos. El control de estos convertidores es complejo, teniendo una respuesta dinámica lenta a los impactos de carga. Hoy en día para asegurar la operación con cargas altamente no lineales y demandas de cargas asimétricas y/o monofásicas son cuestiones obligatorias para ser logrado. En $[1,2]$, tres topologías diferentes (rectificador de 12 pulsos, rectificador trifásico de tres niveles y dos niveles) se han comparado para aplicaciones de futuros aviones más eléctricos. La comparación se realizó en términos de volumen, peso, densidad de potencia, pérdidas de conmutación y eficiencia. Esta comparación mostró que el sistema activo representa la promesa de un incremento de la densidad de potencia con mayor frecuencia de conmutación y/o reducción del volumen de dispositivos pasivos, y en particular, el rectificador de tres niveles se considera la solución más adecuada. Para mejorar el rendimiento del equipo, se han presentado varias técnicas de control. Entre ellas es posible encontrar el control de modo deslizante, control armónico selectivo, control de tiempo muerto (DB), control repetitivo, control proporcional integral (PI), control de espacio de estado óptimo, control proporcional resonante (PR), control de alimentación de avance, entre otros [3-7]. En [8] se presenta la configuración clásica para una GPU que está formada por un convertidor CA-CC no controlado con un convertidor CC-CA controlado. Solo se muestran los resultados de simulación con un esquema de modulación de vectores espaciales para el lado del inversor. Un método dual-loop proporcional-resonante (DLPR) se presenta en [7]. La técnica tiene la ventaja de restringir la perturbación de carga con respuesta dinámica rápida. En [9] se presenta un controlador resonante para el funcionamiento de una unidad de potencia en tierra de $400[\mathrm{~Hz}]$ considerando el cálculo de retardo para mantener un THD reducido de la tensión de salida. En [10] se introduce una nueva estrategia de control utilizando controladores resonantes para la operación en paralelo de convertidores de $400[\mathrm{~Hz}]$ en unidades de potencia de tierra (GPU) para aeronaves. Se requiere operación paralela cuando un solo equipo no puede cumplir con los requisitos de potencia. Se observa que la fase, frecuencia y amplitud de las tensiones de salida en los inversores deben controlarse cuidadosamente (especialmente cuando se utilizan controladores resonantes) porque en caso de una diferencia de fase reducida entre los inversores paralelos podría generar una 
enorme corriente circulante. La estrategia de control propuesta conserva los beneficios del uso de controladores resonantes y un buen rendimiento para compartir la potencia incluso con una pequeña impedancia [11-14]. En [15], se presenta un bucle de tensión individual con controlador de armónicos para obtener una mejor forma de onda del voltaje de salida para tres inversores puente $\mathrm{H}$ monofásicos con transformador de salida de múltiples ramas con conexión Y-Y. Un método de control digital se propone en [16] para mitigar la perturbación de la carga manteniendo un THD bajo, compensando el retraso computacional. La técnica utiliza controladores resonantes diseñados como filtros IIR para eliminar los armónicos $3^{\text {ro }}, 5^{\text {to }}$ y $7^{m o}$. Todos estos los documentos incluyen solo el control del inversor, pero el rectificador y el transformador no se discuten en profundidad. En [17] se utiliza un convertidor matricial ACA-CA monofásico (SPMC) para obtener una forma de onda de salida constante de 100[V]@400[Hz] a pesar de las perturbaciones en la carga o suministro. Los esquemas de modulación clásicos para este convertidor son modulación de ancho pulsos sinusoidales (SPWM) y modulación de ancho de pulso (PWM) minimizando la distorsión armónica. El diseño, así como el control y la implementación de una GPU trifásica para mantenimiento de aeronaves se presenta en [18, 19] utilizando un convertidor matricial trifásico en el que se considera un filtro $L C$ de salida en el lado de salida. Se propone utilizar un convertidor matricial de cuatro piernas con un filtro $L C$ de salida como una unidad de potencia a tierra para aeronave (AGPU) de $400[\mathrm{~Hz}]$ en $[20,21]$. Se considera un controlador resonante y un controlador de voltaje repetitivo de bucle único para regular el convertidor y así obtener una frecuencia y tensión de carga fija independiente de la carga presente en la red de la aeronave utilizando las técnicas de Venturini Óptima y SVM. Un tema importante que no se ha considerado en estos documentos, es que no hay aislamiento ni consideraciones de filtro EMI previstas.

\section{Propuesta de una Nueva Unidad de ALIMENTACIÓN EN TIERRA PARA APLICACIONES AERONÁUTICAS}

\section{A. Descripción General}

La nueva arquitectura se presenta en la Fig. 1. La topología propuesta corresponde a un rectificador trifásico de CA-CC con un circuito de filtro activo de entrada. Este rectificador está conectado a un convertidor de tres niveles con punto neutro (NPC) conectado a un transformador aislado que genera los requeridos 115[V]@400[Hz].

El rectificador trifásico con circuito de filtro activo de entrada se propuso por primera vez en [22], que consta de dos redes activas.

La red de conformación se implementa con un inversor de medio puente a alta frecuencia de conmutación. En esta red un control de corriente genera la corriente del tercer armónico que se sincroniza con la red de CA. La red de inyección está formada por tres dispositivos de conmutación bidireccionales que trabajan a baja frecuencia y un inductor. Solo uno de ellos debe encenderse en el momento, sin embargo, una pequeña superposición a lo largo de las señales de control es necesaria para evitar sobretensiones debido a la característica inductiva. Con estos interruptores es posible seleccionar la ruta de la corriente del inductor para ser agregado a la fase respectiva, generando una corriente de la red sinusoidal con una reducción en las pérdidas de conducción. El terminal de tensión de salida negativo está conectado a la red a través de un diodo de la mitad inferior del puente del rectificador de diodos. Por lo tanto, no se genera voltaje de modo común con la conmutación. El rectificador de inyección de tercer armónico muestra una baja implementación de esfuerzo pero sin un control de la tensión de salida que se determina directamente por el rectificador puente de diodos que exhibe una forma de onda de seis pulsos en cada ciclo.

\section{B. Modulación del Rectificador con Filtro Activo de Entrada}

El circuito de inyección de corriente se modula a baja frecuencia, siguiendo los voltajes de entrada del rectificador considerando que la inyección de corriente activa ocurre en cualquier momento en una sola fase de red basada en la Tabla I. Los interruptores bidireccionales del circuito de inyección conecta el inductor del filtro a la fase de entrada con el valor absoluto más bajo, lo que permite la operación con poca potencia y pérdidas. La magnitud de esta corriente de inyección se define por la potencia de salida del convertidor. Cuando el filtro activo de entrada está deshabilitado, las corrientes de entrada fluyen solo a través del puente de diodos y están altamente distorsionadas. Cuando el filtro activo de entrada está habilitado, la corriente del inductor $i_{L}$ se inyecta a través de los interruptores bidireccionales, logrando corrientes de entrada sinusoidales.

TABLE I: Modulación del circuito de inyección de corriente

\begin{tabular}{cccc} 
Sector & $S_{a}$ & $S_{b}$ & $S_{c}$ \\
\hline \hline $0^{\circ}-60^{\circ}$ & 0 & 1 & 0 \\
$60^{\circ}-120^{\circ}$ & 1 & 0 & 0 \\
$120^{\circ}-180^{\circ}$ & 0 & 0 & 1 \\
$180^{\circ}-240^{\circ}$ & 0 & 1 & 0 \\
$240^{\circ}-300^{\circ}$ & 1 & 0 & 0 \\
$300^{\circ}-360^{\circ}$ & 0 & 1 & 0 \\
\hline \hline
\end{tabular}

Los interruptores del medio puente operan a alta frecuencia de conmutación. Están conectados al otro extremo del inductor, se utilizan para controlar la corriente del inductor de filtro activo de entrada $i_{L}$. Con esta entrada de corriente del inductor de filtro activo $i_{L}$, las corrientes de fuente presentan una forma de onda sinusoidal y en fase con su voltaje fuente respectivo.

\section{Diseño del Inductor}

El valor de la inductancia del convertidor propuesto está diseñada a la frecuencia de conmutación de la red de conformación, considerando:

$$
L \leq \frac{\sqrt{3} \times v_{a, r m s}}{4 \times f_{s} \times \Delta i}
$$

Donde $v_{a, r m s}$ es el valor del voltaje rms, $f_{s}$ es la frecuencia de conmutación, y $\Delta i$ es el ripple de corriente deseado. 


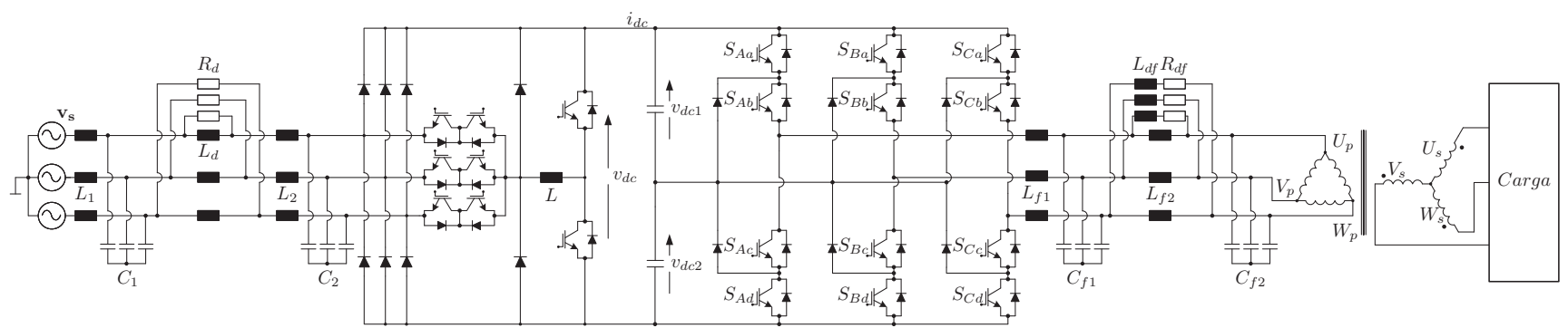

Fig. 1: Topología propuesta para una nueva unidad de alimentación en tierra para aplicaciones aeronáuticas

Al considerar un $\Delta i=25 \%$ del máximo de la corriente que circula por el inductor $i_{L y, \max }$, donde $i_{L y, \max }=I_{N} / 2$, con $I_{N}$ la amplitud de la corriente de entrada $\left(I_{N}=P / V_{N}\right)$.

Para una potencia $P=10[\mathrm{~kW}], V_{N}=480[\mathrm{~V}], \Delta i=$ 2.6[A] y una frecuencia de conmutación $f_{s}=36[\mathrm{kHz}]$, el valor del inductor $L$ está dado como

$$
L \leq \frac{\sqrt{3} \times 480}{4 \times 36000 \times 2.6} \leq 2.2[\mathrm{mH}]
$$

\section{Diseño del Condensador}

El condensador $C$ está diseñado considerando limitar el aumento del voltaje de salida del puente de diodos. Un margen razonable es establecer el ripple de la onda de voltaje máximo $\Delta v$ alrededor del $2.5 \%$ de la tensión de salida del puente de diodos.

$$
C \leq \frac{I_{N}}{2 \times \Delta v \times f_{s}}\left(1-m_{\min }\right),
$$

con $m_{\min }$ el índice de modulación mínimo.

En este trabajo, el ripple máximo de la onda de voltaje se seleccionó como $\Delta v=1.5 \%$ de la tensión de salida del puente de diodo. Por lo tanto,

$$
C \leq \frac{2.6}{2 \times(0.015 \times \sqrt{3} \times 480) \times f_{s}}(1-0.5) \leq 12[\mu F]
$$

Al considerar una conexión en serie de los condensadores, se obtiene

$$
C=\frac{C_{1}+C_{2}}{C_{1} \times C_{2}}[\mu F]
$$

Asumiendo que $C_{1}=C_{2}$, entonces

$$
C=\frac{C_{1}}{2}[\mu F]
$$

\section{E. Modulación para el Inversor NPC}

El estado de salida considera un inversor trifásico de punto neutro (NPC) con un transformador de aislamiento $\Delta$-Y. Para simplificar, el convertidor NPC funciona en lazo abierto. Una compensación por la variación total del enlace CC de voltaje del circuito (forma de seis pulsos) es incluida en el esquema de modulación. Se ha observado que no hay una gran diferencia en el lado de salida incluso cuando la tensión del enlace de voltaje presenta grandes oscilaciones debidas a la inyección de corriente $i_{m}$ a través del punto medio de los condensadores (cuando no se considera minimización de $i_{m}$ ). Este efecto produce grandes variaciones de voltaje que reducen el rango operativo de la tensión de salida y pueden introducir armónicos de baja frecuencia. La modulación del inversor considera la minimización de la corriente del punto medio $i_{m}$ para reducir estas oscilaciones de los voltajes del condensador en el lado de CC.

\section{F. Diseño del Filtro de Salida}

El diseño del filtro de salida debe cumplir las especificaciones con respecto a la calidad de la forma de onda de la tensión de salida, dinámica e interferencia electromagnética conducida (EMI). Según la Fig. 2, se deben cumplir los siguientes requisitos:

- El ripple de la onda de tensión de salida peak-to-peak $\Delta v_{\text {out }}$ por debajo del $1,0 \%$ del valor peak de la tensión de salida nominal, de acuerdo con la norma MIL-STD704F. Esto se define como $\Delta v_{\text {out }} \leq 4.6[\mathrm{~V}]$.

- El ripple de la onda de corriente del inductor de filtro peak-to-peak $\Delta i_{L}$ inferior al $30 \%$ del valor máximo de la corriente de salida nominal. Para este caso $\Delta i_{L} \leq 6.4[\mathrm{~A}]$.

- Máxima impedancia de salida de pequeña señal $Z_{\text {out_max }}$. Se selecciona como 3.5 veces mayor la impedancia de salida máxima, $Z_{\text {out_max }} \leq 3.5[\Omega]$.

- Cumplir con el estándar de acuerdo con MIL-STD-704F para el nivel de emisión conducida.

- Además, el filtro de salida debe tener el peso mínimo, mínimo valor, mínimo costo y/o mínimas pérdidas.

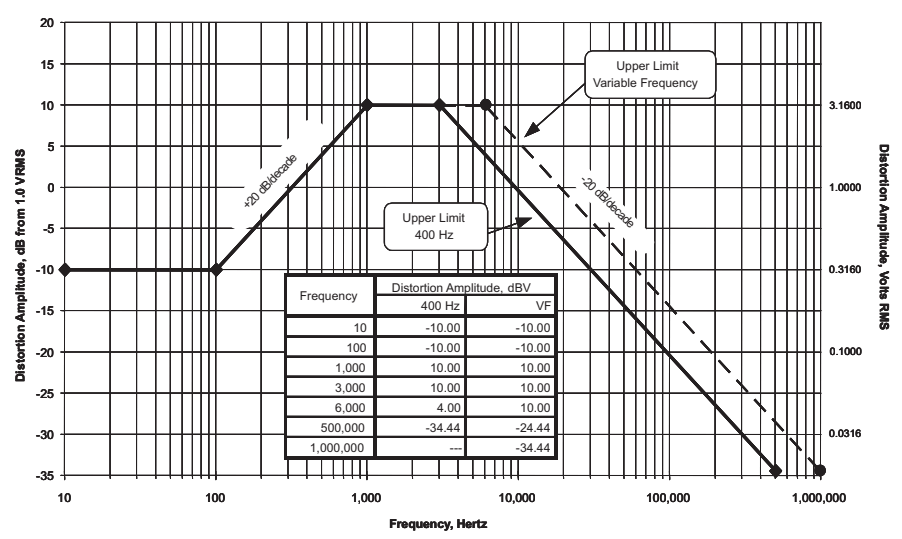

Fig. 2: Espectro de distorsión máxima de 400[Hz] 
El concepto de diseño del filtro de salida es interpretar los requisitos mencionados anteriormente en los límites del plano $L_{f}-C_{f}$. Otras restricciones como la corriente máxima del condensador del filtro reactivo se pueden considerar en el plano $L_{f}-C_{f}$. Siguiendo las pautas presentadas en [23], es posible obtener los parámetros óptimos para el filtro y también para evaluar las ventajas prometedoras de varias topologías de filtro (filtros de una etapa o de varias etapas).

Como se demuestra en [23], no hay espacio de diseño en un filtro de salida de una sola etapa en función de los requisitos mencionados, porque los otros requisitos no se pueden cumplir al mismo tiempo para el criterio EMI. Para resolver este problema, hay al menos cuatro alternativas principales para definir un espacio de diseño común:

1) Diversas piernas de puente por fase podrían ser intercaladas y vinculadas en paralelo a un filtro de una sola etapa.

2) Es posible aumentar los números de niveles de voltaje para disminuir el ripple de la onda de voltaje de salida de alta frecuencia.

3) Diversas piernas de puente por fase se pueden vincular y operar de forma dura en paralelo a un filtro de una sola etapa.

4) Se pueden aumentar el número de etapas del filtro de salida para obtener una mayor atenuación para componentes de alta frecuencia $(>150[\mathrm{kHz}])$ en el voltaje de salida.

Para ahorrar volumen y costo, la opción 4 es seleccionada considerando un filtro de salida de dos etapas. $n$ y $k$ son pesos de escalamiento de la segunda etapa del filtro los que se pueden elegir para obtener el volumen de filtro total más pequeño. Basado en el análisis hecho en [23] (que se hizo usando información comercial), el volumen más pequeño del filtro es $V_{f, \min }=209.2 \mathrm{~cm}^{3}$ y es obtenido para $n=0.01$ y $k=0.9$. Como se representa en la Fig. 1, para evitar resonancias producidas en la segunda etapa de filtro, se agrega un filtro de amortiguación $R_{d}-L_{d}$ paralelo. $L_{d}$ se selecciona como $L_{d}=n L_{f} / 2$.

El ripple máximo de la tensión de salida viene dado por la ec. (7).

$$
\Delta v_{\text {out }}=\frac{m(1-m) v_{d c}}{16 L_{f} C_{f} f_{s}^{2}} \leq 4.6[V]
$$

donde $m$ corresponde al índice de modulación y $f_{s}$ es la frecuencia de conmutación establecida como $f_{s}=36[\mathrm{kHz}]$. Como se observa, $\Delta v_{\text {out }}$ es equivalente al voltaje de enlace $\mathrm{CC}$ $v_{d c}$ e inversamente equivalente a la frecuencia de conmutación.

El ripple máximo de corriente del inductor puede ser determinado como:

$$
\Delta i_{L}=\frac{m(1-m) v_{d c}}{2 L_{f} f_{s}} \leq 6.4[A]
$$

Se observa que esta expresión no depende del valor de capacitancia del filtro $C_{f}$. El ripple de corriente $\Delta i_{L}$ es equivalente al voltaje de enlace $\mathrm{CC} v_{d c}$ e inversamente equivalente a la frecuencia de conmutación.

La máxima impedancia de salida se define como:

$$
Z_{\text {out }}=\frac{L_{f}}{2(1-m) C_{f} Z_{\text {in }}} \leq 3.5[\Omega]
$$

donde $Z_{\text {in }}=v_{d c} /\left(2 \Delta i_{\text {out }}\right)$. La impedancia de salida es linealmente equivalente a la relación a lo largo de la inductancia del filtro y capacitancia del filtro $L_{f} / C_{f}$.

Además, la emisión conducida se considera en base a la Fig. 2, donde se presenta el espectro de distorsión máximo de un sistema 115[V]@400[Hz] basado en el estándar MILSTD-704F [24].

Finalmente, se considera una corriente máxima del condensador del filtro reactivo donde se define el valor máximo de la corriente reactiva por

$$
i_{c, \max }=w_{o} C_{f} \sqrt{2} v_{\text {out }, n} \leq 3[A]
$$

con $w_{o}=2 \pi 400$.

Al usar una frecuencia de conmutación de $f_{s}=36[\mathrm{kHz}]$, es posible obtener los siguientes parámetros que logran elos diferentes criterios de diseño:

- $L_{f 1}=574[\mu \mathrm{H}]$

- $C_{f 1}=3.58[\mu \mathrm{F}]$

- $L_{f 2}=5.74[\mu \mathrm{H}]$

- $C_{f 2}=3.22[\mu \mathrm{F}]$

- $L_{d}=2.87[\mu \mathrm{H}]$

La resistencia de amortiguación $R_{d}$ se obtiene en base a [25]. La selección de $R_{d}$ que reduce la impedancia de salida máxima, para una selección dada de $L_{d}$ es detallada por:

$$
R_{d}=R_{o} Q_{o p t}
$$

donde

$$
\begin{gathered}
R_{o}=\sqrt{\frac{L_{f 2}}{C_{f 2}}} \\
Q_{o p t}=\sqrt{\frac{r(3+4 r)(1+2 r)}{2(1+4 r)}}
\end{gathered}
$$

con $r=L_{d} / L_{f 1}$. Por lo tanto $R_{d}=1.21 \Omega$.

\section{G. Transformador de Aislación}

El filtro de salida está conectado a un transformador de aislación $\Delta$-Y. La razón de la conexión $\Delta$-Y es porque con esta conexión es posible mitigar el efecto de los desequilibrios de carga y las distorsiones.

$$
\begin{gathered}
\frac{N_{p}}{N_{s}}=a \\
V_{s}=\frac{\sqrt{3} v_{o}}{a}
\end{gathered}
$$

\section{RESUltados de Simulación}

Para validar el rendimiento de la topología propuesta y el diseño de los componentes del convertidor, se han hecho simulaciones en el software Gecko Simulation. Se consideran tres casos: primero cuando no hay filtro de entrada y no hay circuito de inyección considerados; segundo, cuando se evalúa la operación con un circuito de inyección y sin filtro de entrada y finalmente, cuando ambos, el filtro de entrada y circuito de inyección están incluidos. 

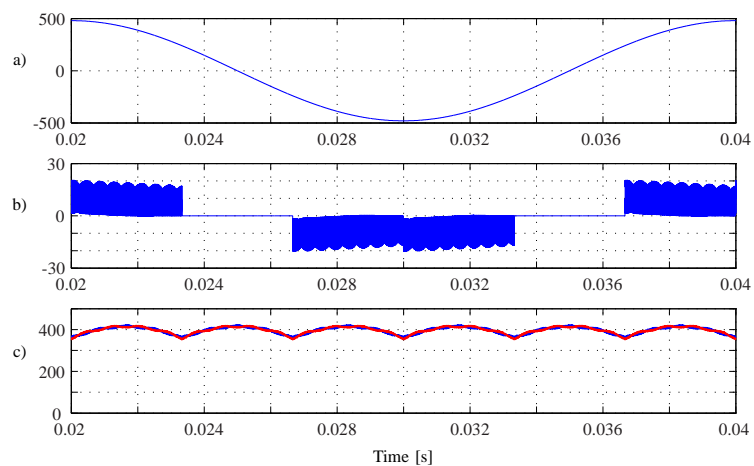

Fig. 3: Resultados de simulación sin filtro de entrada y circuito de inyección: a) voltaje de fuente $v_{s A}[\mathrm{~V}]$; b) corriente de fuente $\left.i_{s A}[\mathrm{~A}] ; \mathrm{c}\right)$ voltaje del condensador $v_{d c 1}[\mathrm{~V}], v_{d c 2}[\mathrm{~V}]$.

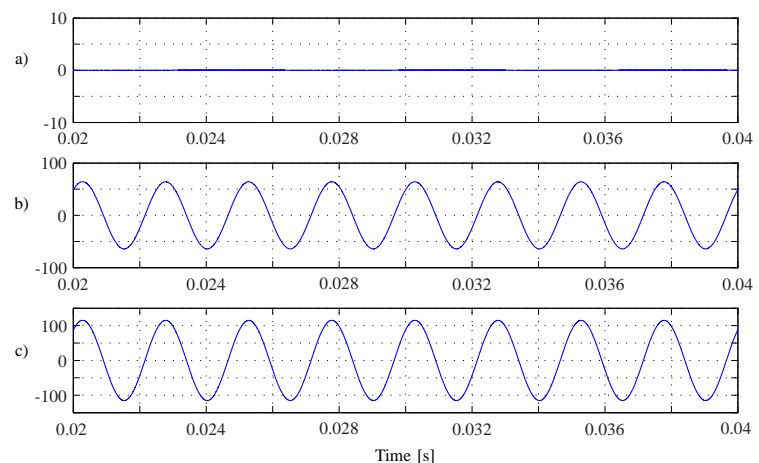

Fig. 4: Resultados de simulación sin filtro de entrada y circuito de inyección: a) corriente IAF $i_{L}[\mathrm{~A}]$; b) corriente de carga $i_{u}[\mathrm{~A}]$; c) voltaje de carga $v_{u}[\mathrm{~V}]$.

En la Fig. 3 y la Fig. 4, se muestran los resultados para el primer caso. En la Fig. 3 es evidente que cuando no hay filtro de entrada ni circuito de inyección considerados, las corrientes de fuente $i_{s A}$ están altamente distorsionadas, lo que es un efecto no deseado (Fig. 3b). Un buen equilibrio de los condensadores del enlace CC $v_{d c 1}$ y $v_{d c 2}$ se observa en la Fig. 3c. En el lado de la carga, la corriente de carga $i_{u}$ y el voltaje de carga $v_{u}$ muestran una forma de onda sinusoidal, logrando la tensión deseada que se ha establecido como 115[V]@400[Hz] (Fig. 4). El rendimiento del convertidor propuesto cuando se considera el circuito de inyección pero no con un filtro de entrada es representado en la Fig. 5 y la Fig. 6. Como era de esperar, la corriente de fuente $i_{s A}$ presenta una forma de onda sinusoidal pero altamente distorsionada (Fig. $5 b)$. De forma similar al caso anterior, se observa un buen equilibrio de los condensadores del enlace CC $v_{d c 1}$ y $v_{d c 2}$ en la Fig. 5c. Se obtiene una forma de onda casi triangular en la corriente del inductor que circula a través del circuito de inyección como se indica en Fig. 6a, que es necesaria para imponer una forma de onda sinusoidal en los terminales de entrada. De nuevo, la corriente de carga $i_{u}$ y tensión de carga $v_{u}$ muestran una forma de onda sinusoidal logrando la tensión deseada que se ha establecido como 115[V]@400[Hz] (Fig. 6b y Fig. 6c). Finalmente, en la Fig. 7 y la Fig. 8 se muestran los resultados de simulación del convertidor propuesto, que
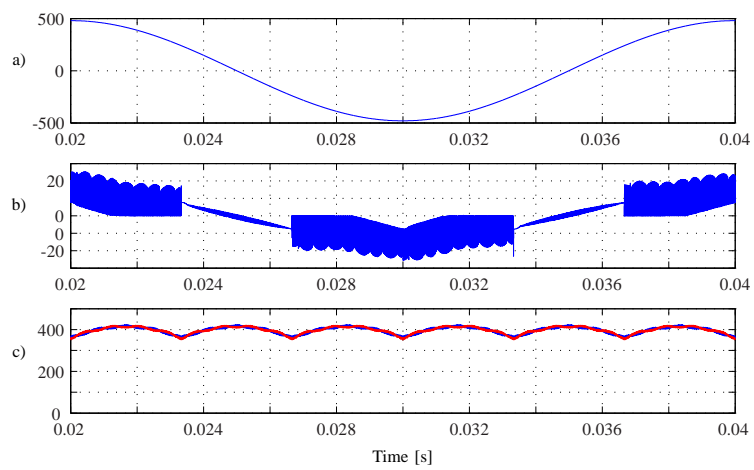

Fig. 5: Resultados de simulación sin filtro de entrada: a) voltaje de fuente $v_{s A}[\mathrm{~V}] ;$ b) corriente de fuente $i_{s A}[\mathrm{~A}] ;$ c) voltaje del condensador $v_{d c 1}[\mathrm{~V}], v_{d c 2}[\mathrm{~V}]$.
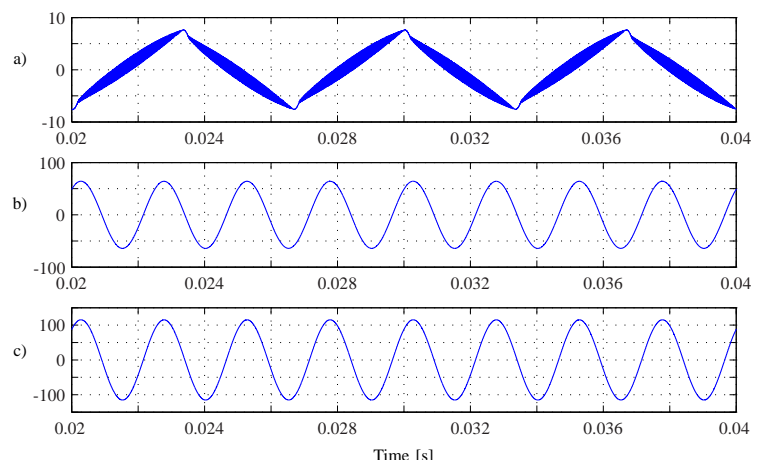

Fig. 6: Resultados de simulación sin filtro de entrada: a) corriente IAF $i_{L}[\mathrm{~A}]$; b) corriente de carga $i_{u}[\mathrm{~A}]$; c) voltaje de carga $v_{u}[\mathrm{~V}]$.

funciona con el filtro de entrada y el circuito de inyección de corriente. Como se esperaba, el rendimiento de la topología propuesta produce una corriente de fuente sinusoidal $i_{s A}$ con una baja distorsión armónica debido al circuito de inyección y al filtro de entrada. De manera similar a los dos casos anteriores, los condensadores del enlace CC $v_{d c 1}$ y $v_{d c 2}$ se mantienen equilibrados todo el tiempo, Fig. 7c. En la Fig. 8a se observa la corriente del inductor a través del circuito de inyección. Se observa la corriente de carga sinusoidal $i_{u}$ y la tensión $v_{u}$ Fig. 8.

\section{CONCLUSIÓN}

En este documento se ha presentado una nueva topología CA-CA para ser usada como suministro de alimentación en tierra para aplicaciones aeronáuticas. El convertidor propuesto se basa en un convertidor de CA-CC con un circuito de inyección de corriente vinculado con un convertidor de tres niveles conectado a un transformador de aislación para generar 115[V]@400[Hz]. El diseño de los diferentes los componentes que crean el convertidor están detallados. Los resultados de la simulación han demostrado el correcto desempeño del convertidor.

\section{AgRADECIMIENTOS}

Los autores agradecen al Projecto FONDECYT Regular 1160690 y del Proyecto MEC 80150056. 

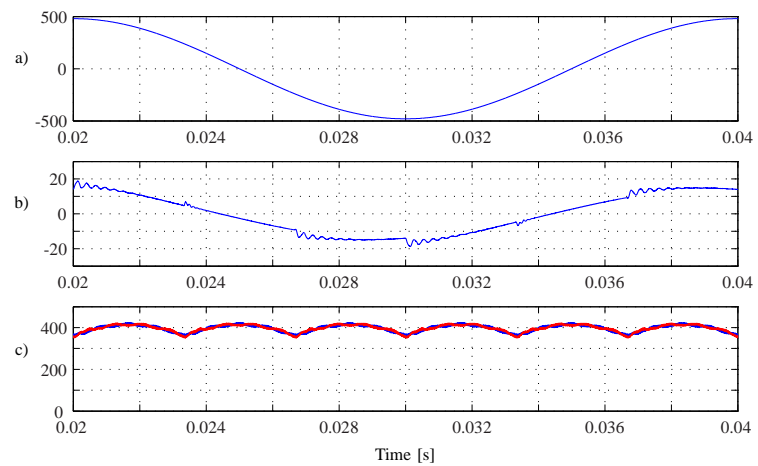

Fig. 7: Resultados de simulación sin filtro de entrada: a) voltaje de fuente $v_{s A}[\mathrm{~V}]$; b) corriente de fuente $i_{s A}[\mathrm{~A}]$; c) voltaje del condensador $v_{d c 1}[\mathrm{~V}], v_{d c 2}[\mathrm{~V}]$.
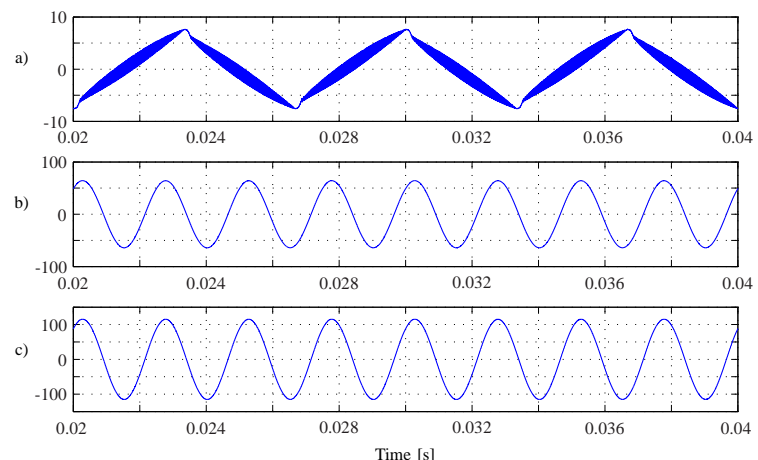

Fig. 8: Resultados de la simulación: a) corriente IAF $i_{L}[\mathrm{~A}]$; b) corriente de carga $i_{u}[\mathrm{~A}]$; c) votaje de carga $v_{u}[\mathrm{~V}]$.

\section{REFERENCIAS}

[1] G. Gong, M. Heldwein, U. Drofenik, K. Mino, and J. Kolar, "Comparative evaluation of three-phase high power factor ac-dc converter concepts for application in future more electric aircrafts," in Applied Power Electronics Conference and Exposition, 2004. APEC '04. Nineteenth Annual IEEE, vol. 2, 2004, pp. 1152-1159 vol.2.

[2] G. Gong, M. Heldwein, U. Drofenik, J. Minibock, K. Mino, and J. Kolar, "Comparative evaluation of three-phase high-power-factor acdc converter concepts for application in future more electric aircraft," Industrial Electronics, IEEE Transactions on, vol. 52, no. 3, pp. 727737, 2005.

[3] U. Jensen, M. Rasmussen, T. Mortensen, F. Blaabjerg, and J. Pedersen, "A new control method for $400 \mathrm{hz}$ ground power units for airplanes," in Industry Applications Conference, 1998. Thirty-Third IAS Annual Meeting. The 1998 IEEE, vol. 2, 1998, pp. 1469-1476 vol.2.

[4] U. Jensen, F. Blaabjerg, and J. Pedersen, "A new control method for 400-hz ground power units for airplanes," Industry Applications, IEEE Transactions on, vol. 36, no. 1, pp. 180-187, 2000.

[5] L. Mihalache, "Dsp control of $400 \mathrm{hz}$ inverters for aircraft applications," in Industry Applications Conference, 2002. 37th IAS Annual Meeting. Conference Record of the, vol. 3, 2002, pp. 1564-1571 vol.3.

[6] Z. Housheng, Z. Yanlei, and L. Haidong, "Design of three-phase intermediate frequency aviation power based on single chip microcomputer," in Computer Engineering and Technology (ICCET), 2010 2nd International Conference on, vol. 4, 2010, pp. V4-669-V4-672.

[8] G. Basile, S. Buso, S. Fasolo, P. Tenti, and P. Tomasin, "A 400 hz, 100 kva, digitally controlled ups for airport installations," in Industry Applications Conference, 2000. Conference Record of the 2000 IEEE, vol. 4, 2000, pp. 2261-2268 vol.4.
[7] J. Zhu, Z. Nie, W. Ma, and S. Nie, "Comparison between db control and dual-loop pr control for collapsed h-bridge single-phase $400 \mathrm{hz}$ power supply," in Industrial Electronics (ISIE), 2012 IEEE International Symposium on, 2012, pp. 240-245.

[9] Z. Li, P. Wang, H. Zhu, Y. Li, L. Tan, Y. Chen, and F. Gao, "A new digital control method for high performance $400 \mathrm{hz}$ ground power unit," in Power Electronics and Motion Control Conference, 2008. EPE-PEMC 2008. 13th, 2008, pp. 515-520.

[10] F. Gao, P. Wang, Y. Li, Z. Li, and H. Zhu, "Wireless parallel operation of 400-hz high-power inverters in ground power units for airplanes," in Power Electronics and Motion Control Conference (EPE/PEMC), 2010 14th International, 2010, pp. T3-165-T3-171.

[11] Z. Li, Y. Li, P. Wang, H. Zhu, C. Liu, and F. Gao, "Single-loop digital control of high-power 400-hz ground power unit for airplanes," Industrial Electronics, IEEE Transactions on, vol. 57, no. 2, pp. 532543, 2010.

[12] L. Chun-xi, M. Wei-ming, S. Chi, and H. Wen-hua, "Research on harmonics suppression in high power middle frequency $400 \mathrm{hz}$ inverter," in Power and Energy Engineering Conference, 2009. APPEEC 2009. Asia-Pacific, 2009, pp. 1-4.

[13] C. Liu, W. Ma, C. Sun, and W. Hu, "Research on digital control design of high power middle frequency $400 \mathrm{hz}$ inverter power," in Power Electronics and Motion Control Conference, 2009. IPEMC '09. IEEE 6th International, 2009, pp. 1544-1549.

[14] C. Liu, C. Sun, and W. Hu, "Proportional-resonant controller of high power $400 \mathrm{hz}$ inverter in stationary frame," in Electrical Machines and Systems, 2008. ICEMS 2008. International Conference on, 2008, pp. 1772-1777.

[15] H. Zhu, Y. Li, Z. Li, and P. Wang, "Design and performance analysis of high power static 400-hz supply," in Power and Energy Engineering Conference, 2009. APPEEC 2009. Asia-Pacific, 2009, pp. 1-4.

[16] L. Mihalache, "Improved load disturbance rejection method for $400 \mathrm{hz}$ gpu inverters," in Applied Power Electronics Conference and Exposition, 2004. APEC '04. Nineteenth Annual IEEE, vol. 1, 2004, pp. 95-101 Vol.1.

[17] G. Mahalakshmi, A. Jamna, and V. Jamuna, "A single phase matrix converter for agpu," in Computing, Electronics and Electrical Technologies (ICCEET), 2012 International Conference on, 2012, pp. 224-231.

[18] S. Lopez Arevalo, P. Zanchetta, P. Wheeler, A. Trentin, and L. Empringham, "Control and implementation of a matrix-converter-based ac ground power-supply unit for aircraft servicing," Industrial Electronics, IEEE Transactions on, vol. 57, no. 6, pp. 2076-2084, 2010.

[19] S. Arevalo, P. Zanchetta, and P. Wheeler, "Control of a matrix converterbased ac power supply for aircrafts under unbalanced conditions," in Industrial Electronics Society, 2007. IECON 2007. 33rd Annual Conference of the IEEE, 2007, pp. 1823-1828.

[20] W. Rohouma, L. Empringham, P. Zanchetta, and P. Wheeler, "A four legs matrix converter based ground power unit with selective harmonic control," in Energy Conversion Congress and Exposition (ECCE), 2011 IEEE, 2011, pp. 799-805.

[21] W. Rohouma, L. de Lillo, S. Lopez, P. Zanchetta, and P. Wheeler, "A single loop repetitive voltage controller for a four legs matrix converter ground power unit," in Power Electronics and Applications (EPE 2011), Proceedings of the 2011-14th European Conference on, 2011, pp. 1-9.

[22] N. Vazquez, H. Rodriguez, C. Hernandez, E. Rodriguez, and J. Arau, "Three-phase rectifier with active current injection and high efficiency," Industrial Electronics, IEEE Transactions on, vol. 56, no. 1, pp. 110119, 2009.

[23] D. Boillat, T. Friedli, J. Muhlethaler, J. Kolar, and W. Hribernik, "Analysis of the design space of single-stage and two-stage lc output filters of switched-mode ac power sources," in Power and Energy Conference at Illinois (PECI), 2012 IEEE, Feb 2012, pp. 1-8.

[24] Y. Shanshui, L. Yan, Z. Jian, C. Mengdi, and W. Li, "Research on methods of distortion spectrum analysis for aircraft vfac power system," in Power Electronics and Applications (EPE), 2013 15th European Conference on, Sept 2013, pp. 1-8.

[25] R. Erickson, "Optimal single resistors damping of input filters," in Applied Power Electronics Conference and Exposition, 1999. APEC '99. Fourteenth Annual, vol. 2, Mar 1999, pp. 1073-1079 vol.2. 